\title{
Numerical Investigation of Scattering from a Surface Dielectric Barrier Discharge Actuator under Atmospheric Pressure
}

\author{
Yuna $\mathrm{Kim}^{1} \cdot$ Sangin Kim${ }^{1} \cdot$ Doo-Soo Kim${ }^{2} \cdot$ Il-Young $\mathrm{Oh}^{3} \cdot$ Jong-Gwan Yook ${ }^{1, *}$
}

\begin{abstract}
Surface dielectric barrier discharge (SDBD), which is widely used to control turbulence in aerodynamics, has a significant effect on the radar cross-section (RCS). A four-way linearly synthesized SDBD air plasma actuator is designed to bolster the plasma effects on electromagnetic waves. The diffraction angle is calculated to predict the RCS because of the periodic structure of staggered electrodes. The simplified plasma modeling is utilized to calculate the inhomogeneous surface plasma distribution. Monostatic RCS shows the diffraction in the plane perpendicular to the electrode array and the notable distortion by plasma. In comparison, the overall pattern is maintained in the parallel plane with minor plasma effects. The trends also appear in the bistatic RCS, which has a significant difference in the observation plane perpendicular to the electrodes. The peaks by Bragg's diffraction are shown, and the RCS is reduced by $10 \mathrm{~dB}$ in a certain range by the plasma effect. The diffraction caused by the actuator and the inhomogeneous air plasma should be considered in designing an SDBD actuator for a wide range of application.
\end{abstract}

Key Words: Actuator, Diffraction, Plasma, Radar Cross-Section, Surface Dielectric Barrier Discharge.

\section{INTRODUCTION}

A surface dielectric barrier discharge (SDBD) actuator has been examined as a flow controller in aerodynamics [1]. It has several advantages, such as switchable plasma property, absence of mechanical movement, and simple structure, among others. Several efforts have been made to utilize the actuator in flying vehicles. In some studies, SDBD is attached to turbine blades [2, $3]$, and it presents an outstanding performance in the control of flow separation. Other studies use the actuator to change the air flow around the airfoil [4]. The plasma effect as the change in the angle of flow attack is demonstrated in [5]. At the other end of the spectrum, researchers have attempted to enhance the plasma effect by enlarging the plasma area. Linearly synthesized SDBD has been proposed [6], and it generates a large plasma area. The sliding plasma discharge is examined by designing the electrodes properly [7].

Accordingly, the plasma effects on electromagnetics have gained the interest of researchers because the scattered waves can be influenced by the staggered electrode structures. In addition, the incident waves and plasma may possibly interact. Therefore, the radar cross-section (RCS) of flying vehicles,

\footnotetext{
Manuscript received August 28, 2017 ; Revised November 7, 2017 ; Accepted December 24, 2017. (ID No. 20170828-040J)

${ }^{1}$ Department of Electrical and Electronic Engineering, Yonsei University, Seoul, Korea.

${ }^{2}$ Agency for Defense Development, Daejeon, Korea.

${ }^{3}$ School of Electrical Engineering, Dongyang Mirae University, Seoul, Korea.

${ }^{*}$ Corresponding Author: Jong-Gwan Yook (e-mail: jgyook@yonsei.ac.kr)
}

This is an Open-Access article distributed under the terms of the Creative Commons Attribution Non-Commercial License (http://creativecommons.org/licenses/by-nc/4.0) which permits unrestricted non-commercial use, distribution, and reproduction in any medium, provided the original work is properly cited.

(c) Copyright The Korean Institute of Electromagnetic Engineering and Science. All Rights Reserved. 
which is one of the crucial factors for the survival rate of aircrafts, may change. Unexpected effects on RCS may occur because of the SDBD actuators installed for the purpose of controlling flow. To demonstrate the effect on electromagnetic waves, experiments in a certain observation plane have been made [8]. However, various observation planes are required because the SDBD structure has staggered electrodes that can cause reflection and diffraction. Another study analyzes the diffraction based on simulation under low pressure. It reveals that the RCS increases at a specific angle [9]. Experiments under atmospheric pressure are necessary for flying vehicles. The study [10] based on $2 \mathrm{D}$ simulation should be extended to $3 \mathrm{D}$ to enable analysis in various angles.

The current study focuses on the change in scattering by the SDBD air plasma actuator under atmospheric pressure. Monostatic and bistatic RCS in different observation planes is simulated to demonstrate the effect of a plasma actuator on survivability.

\section{STRUCTURE OF A PLASMA ACTUATOR}

The linearly synthesized SDBD actuator consists of four unit actuators to enhance the plasma effect. The sufficient spacing between the actuators reduces the interference between the adjacent units. Each unit has an exposed and buried electrode with a dielectric barrier. The plasma is generated above the buried electrode (Fig. 1). The plasma actuator is backed by a metal plate to define the surface of the target. The targetis square, with each side measuring $120 \mathrm{~mm}$. The configuration of the designed actuator is illustrated in Fig. 2. The size of the electrode strip is $100 \mathrm{~mm} \times 12 \mathrm{~mm}$. The spacing between two units is $8 \mathrm{~mm}$. A substrate with a permittivity of 2.3 is used as the dielectric barrier.

Fig. 3 shows the air plasma generation of the designed actuator. Clearly, the visible plasma is generated on top of the buried electrodes. In addition, the interaction between the unit SDBD actuators is limited. The color of the plasma indicates that the air plasma is generated.

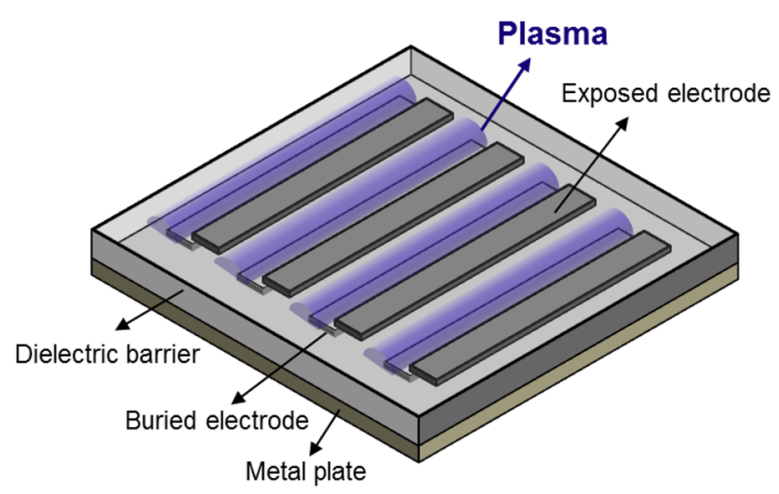

Fig. 1. Structure of the SDBD plasma actuator.

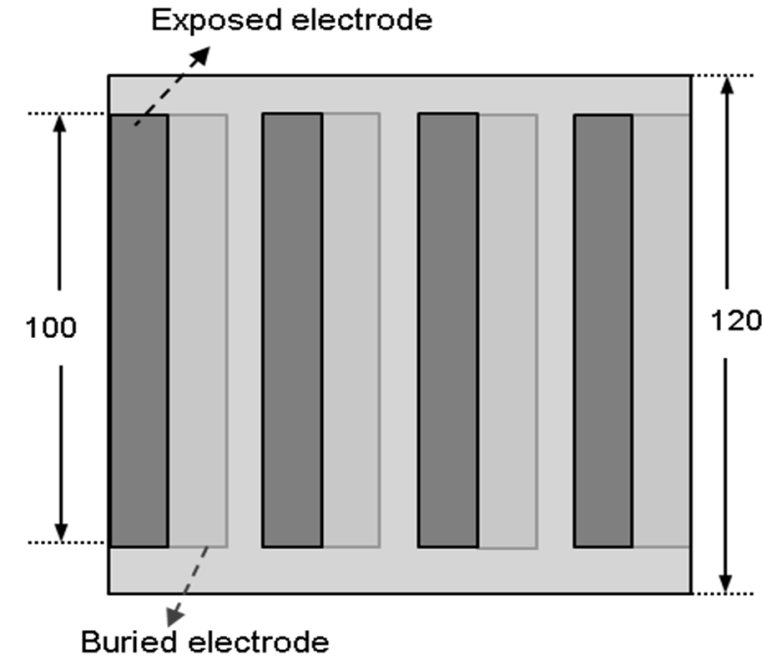

(a) Configuration

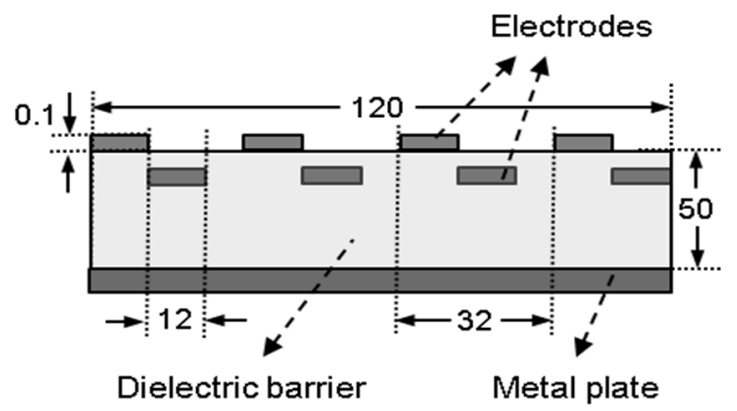

(b) Cross section of the generator

Fig. 2. Configuration of the plasma actuator (unit in $\mathrm{mm}$ ). (a) Configuration. (b) Cross section of the generator.

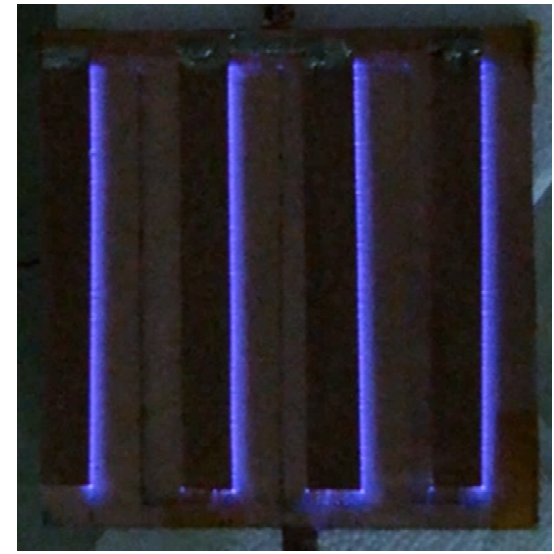

Fig. 3. Generated air plasma from the SDBD discharge actuator.

\section{EFFECT ON ELECTROMAGNETIC WAVES}

\section{Diffraction from the Plasma Actuator}

The SDBD actuator has a periodic structure, which causes a grating diffraction with the conductor strips acting as a crystal [11]. In Eq. (1), the relation between the incident wave and the diffraction wave is predicted when the grating spacing is determined. 


$$
\sin \theta_{d}-\sin \theta_{i}=m \frac{\lambda}{\Lambda},
$$

where $\theta_{d}$ is the diffraction angle, $\theta_{1}$ is the incident angle, $\theta_{i}$ is the grating spacing between two elements, and $m$ is the integer number. With the incident angle of $0^{\circ}$ and $\Lambda$ of $32 \mathrm{~mm}$, the diffraction angle is calculated as $38.7^{\circ}$ when the frequency of the incident wave is $15 \mathrm{GHz}$. Therefore, the maximum occurs at the angle with the bistatic RCS simulation.

The monostatic RCS can be analyzed as the Bragg diffraction:

$$
2 \sin \theta_{d}=m \frac{\lambda}{\Lambda}
$$

The calculation result is $18.2^{\circ}$ when $m$ is 1 . As a result, monostatic RCS has a maximum diffraction at the angle.

\section{Interaction with the Plasma Array}

The interaction between cold plasma and incident electromagnetics is determined by dispersion relation,

$$
k=k^{\prime}-j k^{\prime \prime}=\sqrt{\frac{\omega^{2}}{c^{2}}-\frac{\omega_{p}{ }^{2}}{c^{2}\left(1-j \frac{\nu_{c}}{\omega}\right)}}
$$

where $\omega_{p}$ is the plasma frequency, $v_{c}$ is the collision frequency, and $\omega_{c}$ is the operating frequency of the incident wave [12]. Plasma frequency and collision frequency are highly dependent on the plasma generator structure and the ambient pressure and temperature. The collision frequency is a function of the type of gas, neutral gas density, and electron temperature [13]. Measuring all of these variables is difficult. The value of collision frequency is $2.1 \mathrm{THz}$, which is obtained from the measurement of helium glow discharge under atmospheric pressure [14]. The plasma frequency is calculated from the equation,

$$
\omega_{p}=\sqrt{\frac{n_{e} e^{2}}{m \varepsilon_{0}}}
$$

where $n_{\mathrm{e}}$ is the electron density, $e$ is the electron charge, and $m$ is the mass of the electron. The range of electron density of the DBD actuator is $10^{17}-10^{21} \mathrm{~m}^{-3}[15]$. The electron density largely varies as the structure and the environment change. It is the key to analyzing the interaction between plasma and electromagnetic waves. The electron density and its distribution should be derived. As shown in the following section, plasma modeling for the given actuator provides the electron density distribution.

\section{Plasma MODELING FOR THE ACTUATOR}

To derive plasma modeling for the actuator, a simplified modeling for charged particles in plasma is utilized [15]. The relation between the charged particle and the electric potential is defined as Eq. (5).

$$
\nabla \cdot(-\nabla \Phi)=\frac{\rho_{c}}{\varepsilon}
$$

where $\Phi$ is the electric potential in the conductive fluid, and $\rho_{c}$ is the charge density. In Eq. (6), the electric potential can be separated into electric potential due to electrode $\phi$ and electric potential due to charged particles in plasma $\varphi_{\mathrm{c}}$. Under the assumption that the Debye thickness is small and the charge on the wall is not large, the potential caused by the electric charge is the dominant factor in the decision of the charge distribution. The effect of the external electric field is relatively small [16]. Then, Eq. (5) can be converted to Eq. (7).

$$
\begin{gathered}
\Phi=\phi+\varphi_{c}, \\
\nabla \cdot\left(\varepsilon_{r} \nabla \rho_{c}\right)=-\frac{\rho_{c}}{\lambda_{d}^{2}},
\end{gathered}
$$

where $\varepsilon_{r}$ is the relative permittivity, and $\lambda_{d}$ is the Debye thickness. Based on a time-independent system, the density of the charged particle can be expressed as a normalized from

$$
\rho_{c}^{*}=\frac{\rho_{c}}{\rho_{c}^{\max } f(t)},
$$

where $\rho_{c}^{\max }$ is the maximum charged particle density and $f(t)$ is the time-dependent factor. On the surface of the dielectric, the charged particle follows a half Gaussian distribution [17]. Then, the electron density can be derived as

$$
n_{e}=\rho_{c} \exp \left(\frac{e \Phi}{k_{B} T_{e}}\right)
$$

where $k_{B}$ is the Boltzmann constant, and $T_{e}$ is the electron temperature. The calculated normalized electron density is shown in Fig. 4 by using multi-physics software (COMSOL Inc., Palo Alto, CA, USA). The maximum occurs on top of the buried electrode, and the density weakens as the distance from the surface increases. The maximum electron density is set to $1 \times 10^{21}$ $\mathrm{m}^{-3}$, which corresponds to the electron range in a previous work

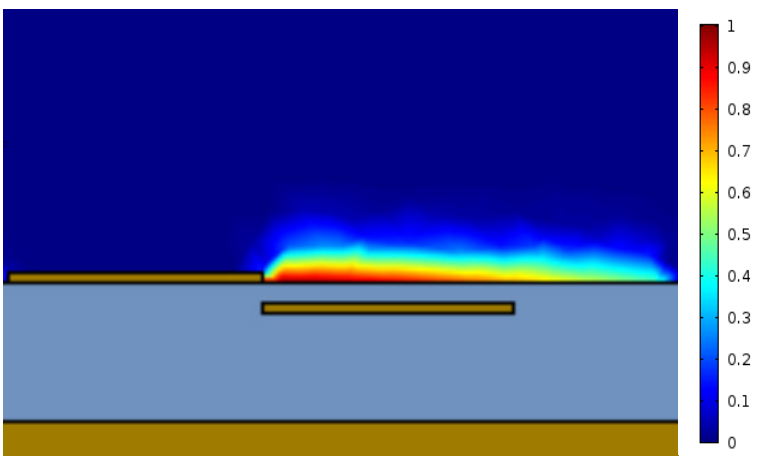

Fig. 4. Normalized charge density distribution, $\rho_{c}^{*}$. 


\section{RCS OF THE TARGET}

Fig. 5 shows the coordinates for the DBD plasma actuator in the $x-y$ plane. Monostatic and bistatic RCSs are calculated as shown in Figs. 6-9 using COMSOL multi-physics software.

\section{Monostatic RCS}

When the linearly polarized electromagnetic wave along the $y$-axis is incident toward the DBD with a frequency of $15 \mathrm{GHz}$, the monostatic RCS is observed in the $x-z$ plane as shown in Fig. 6. The copper plate has a prominent peak at $0^{\circ}$. Most of the field hits the plate and reflects back toward it. If the radar is in the different direction, backward reflection will decrease because of specular reflection. For the plasma-off state, the peak around $0^{\circ}$ is explained similarly. At a different angle, the incident fields are scattered by the electrodes, which increase the level of monostatic RCS. Accordingly, the RCS for the plasmaoff state is higher than that for the copper plate. In addition, the simulated result with the plasma-off is compared with the measured result reported by Wolf and Arjomandi [8]. A good agreement was found in the simulated and measured results in the range of $-20^{\circ}$ to $20^{\circ}$. The main beam at $0^{\circ}$ is observed because of a specular reflection from the copper plate. The experimental result indicated a distorted pattern in $30^{\circ}-40^{\circ}$. The level of measurement result with the plasma-off state is higher than that of the simulation. This outcome may be due to the undesired effects from background noise. However, the simulated monostatic RCS of DBD and the analytic solution of the square copper plate show symmetry. When the plasma is generated, the graph shows no significant change. The generated plasma may have a minor effect on the monostatic RCS in the $\varphi=0^{\circ}$ plane.

However, the trend is completely changed in other observation planes. When $\varphi=90^{\circ}$, the monostatic RCS has an asym[15]. The electron density distribution is used to find the plasma frequency in Eq. (4).

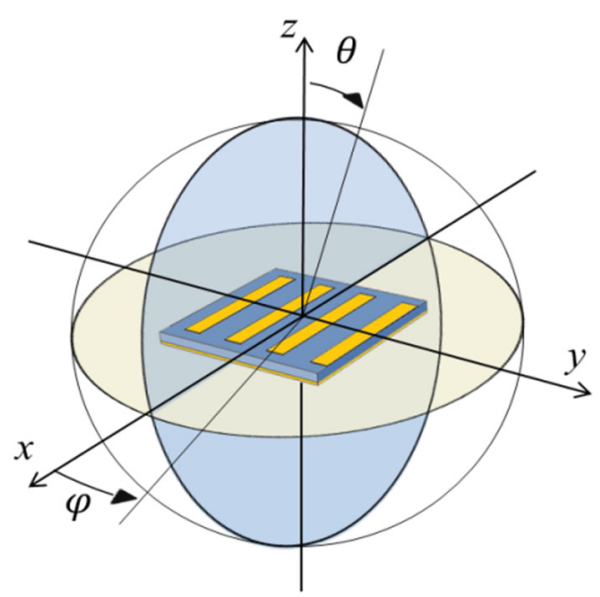

Fig. 5. Coordinates for the dielectric barrier discharge actuator.

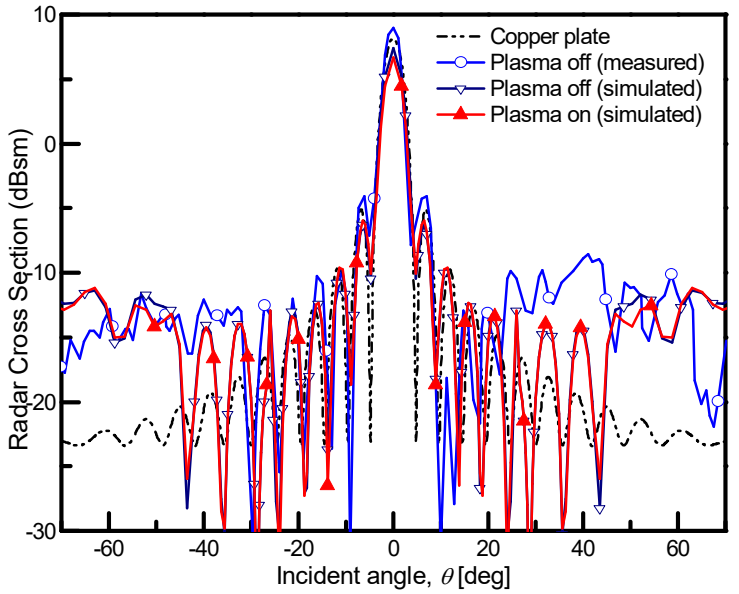

Fig. 6. Monostatic RCS for the incident wave of a $15-\mathrm{GHz}$ frequency when $\varphi=0^{\circ}$.

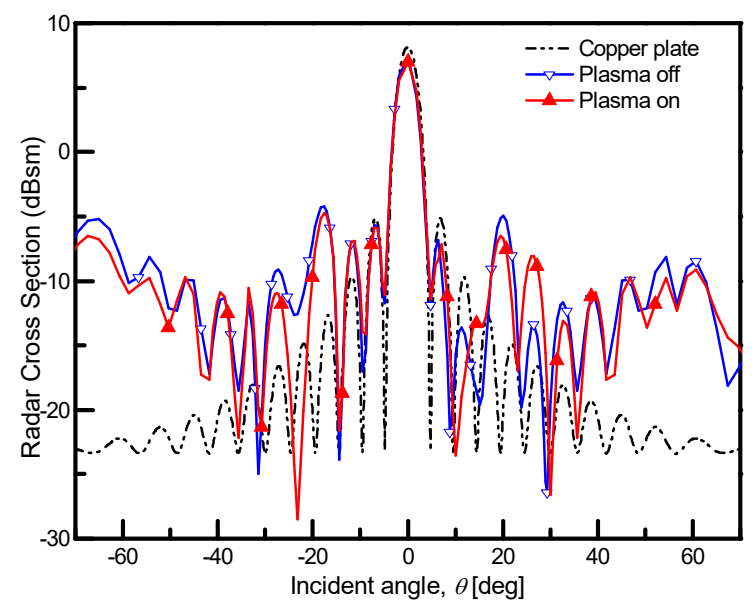

Fig. 7. Monostatic RCS for the incident wave of a $15-\mathrm{GHz}$ frequency when $\varphi=90^{\circ}$.

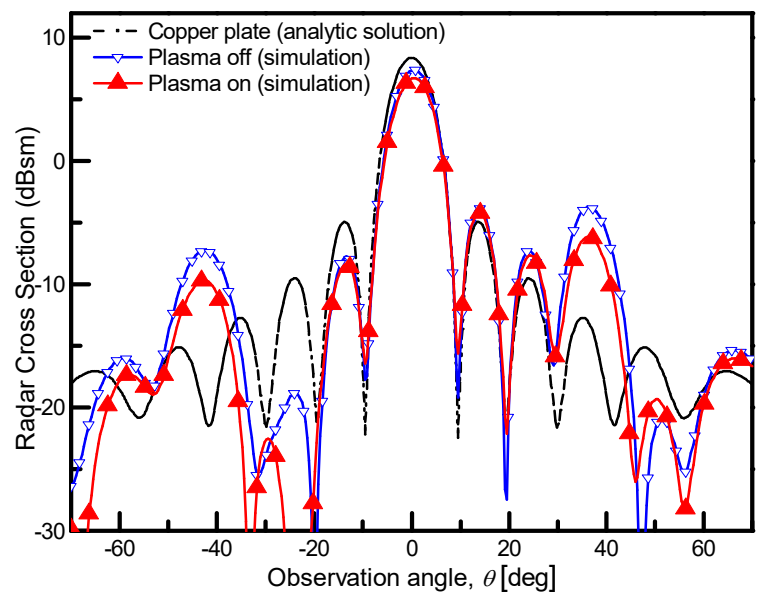

Fig. 8. Bistatic RCS for the incident wave of a $15-\mathrm{GHz}$ frequency with the observation in the $x-z$ plane.

metric pattern in the $y-z$ plane because of the staggered electrodes in the actuator. Compared with the copper plate, sudden 


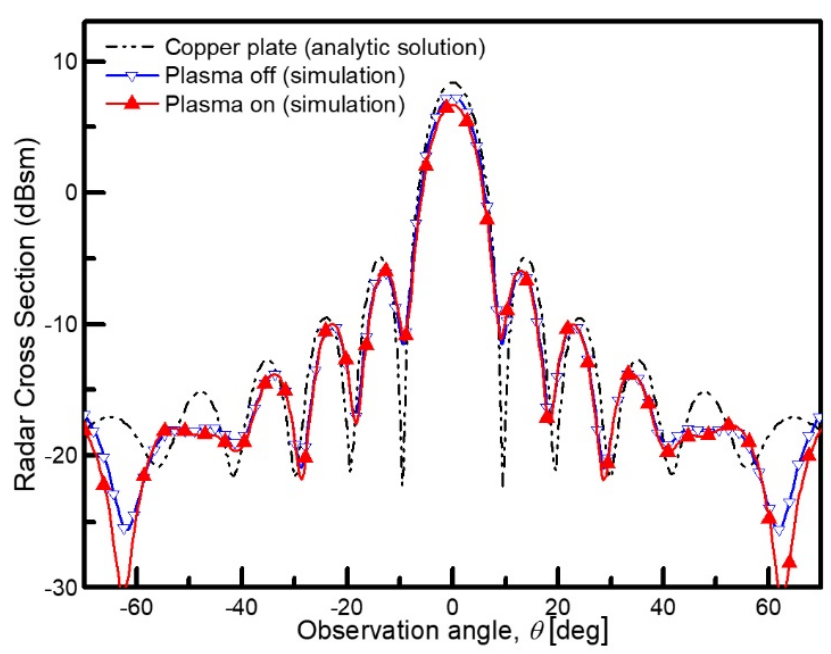

Fig. 9. Bistatic RCS for incident wave of $15 \mathrm{GHz}$ frequency with the observation in $y$ - $z$ plane.

increases occur near $-20^{\circ}$ and $20^{\circ}$ that are caused by the Bragg diffraction, the calculated incident angle of which is $18.2^{\circ}$ in the previous section. When the plasma is generated, a decrease of $10^{\circ}-20^{\circ}$ occurs, and a sudden increase is observed in $30^{\circ}$. The reason for this outcome is that the inhomogeneity of the plasma distribution changes the direction of the scattering waves.

\section{Bistatic RCS}

Fig. 8 shows the bistatic RCS when the incident wave is linearly polarized in the $x-z$ plane. The plasma-off state RCS is comparable with that in the case of the square copper plate. A peak at $0^{\circ}$ is observed because of the specular reflection. In addition, the plasma effect does not appear in the graph. As shown in Fig. 8, the distortion due to the plasma actuator is notable in Fig. 9. When the incident angle is $0^{\circ}$, the diffraction angle is $38.7^{\circ}$. Two peaks due to diffraction can be observed at the diffraction angles. The peaks are at least $5 \mathrm{~dB}$ higher than that in the case of the copper plate. As the plasma is generated, the overall level decreases by $2-3 \mathrm{~dB}$. The RCS near $-25^{\circ}$ has notable effects. The attenuation by plasma decreases the RCS by $10 \mathrm{~dB}$ at the frequency range. It is related to the distribution of plasma, which is periodically located near exposed electrodes. The reduction is caused by both the staggered electrode alignment of the generator and the plasma distribution.

\section{CONCLUSION}

The SDBD plasma has a significant effect on RCS. Peaks by diffraction are observed in both plasma-off and -on states because of the periodicity of staggered electrodes. In the plasmaon state, the inhomogeneous plasma distribution causes both the reduction of the level and the change in scattering angles. These effects become different as the RCS observation plane changes.

If the operating frequency or spacing between electrodes is changed, the location of peaks due to diffraction can be controlled. Therefore, moving the peaks to the unimportant angles is possible, thus reducing the level of RCS in the desired range of angles. However, the changes in the structure affect the process of plasma generation. They lead to different reflections and absorption properties by plasma. Therefore, such relations should be considered in the DBD actuator design. Further research should include the effect of a complex electrode structure on plasma generation.

This work was supported by the Low Observable Technology Research Center Program of the Defense Acquisition Program Administration and the Agency for Defense Development of the Republic of Korea.

\section{REFERENCES}

[1] T. C. Corke, C. L. Enloe, and S. P. Wilkinson, "Dielectric barrier discharge plasma actuators for flow control," Annual Review of Fluid Mechanics, vol. 42, pp. 505-529, 2010.

[2] J. Huang, T. C. Corke, and F. O. Thomas, "Plasma actuators for separation control of low-pressure turbine blades," ALAA Journal, vol. 44, no. 1, pp. 51-57, 2006.

[3] R. Van Dyken, H. Perez-Blanco, A. Byerley, and T. McLaughlin, "Plasma actuator for wake flow control of high camber blades during part load operation," in Proceedings of the ASME Turbo Expo 2004: Power for Land, Sea, and Air, Vienna, Austria, 2004, pp. 351-363.

[4] T. C. Corke, B. Mertz, and M. P. Patel, "Plasma flow control optimized airfoil," in Proceedings of the 4th ALAA Aerospace Sciences Meeting and Exhibit, Reno, NV, 2006, pp. 1-13.

[5] J. R. Roth, "Aerodynamic flow acceleration using paraelectric and peristaltic electrohydrodynamic effects of a one atmosphere uniform glow discharge plasma," Physics of Plasmas, vol. 10, no. 5, pp. 2117-2126, 2003.

[6] J. R. Roth, D. M. Sherman, and S. P. Wilkinson, "Boundary layer flow control with a one atmosphere uniform glow discharge surface plasma," NASA Langley Research Center, Technical Report, 1998.

[7] C. Louste, G. Artana, E. Moreau, and G. Touchard, "Sliding discharge in air at atmospheric pressure: electrical properties," Journal of Electrostatics, vol. 63, no. 6, pp. 615-620, 2005.

[8] S. Wolf and M. Arjomandi, "Investigation of the effect of dielectric barrier discharge plasma actuators on the radar cross section of an object," Journal of Physics D: Applied Physics, vol. 44, no. 31, article ID. 315202, 2011.

[9] F. Mirhosseini, B. G. Colpitts, R. Pimentel, and Y. de Villers, "The effect of dielectric-barrier-discharge plasma actuators on electromagnetic," IEEE Transactions on Plasma Science, vol. 44, 
no. 4, pp. 665-669, 2016.

[10] Y. Kim, I. Y. Oh, J. G. Yook, and Y. Hong, "Numerical investigation of electromagnetic characteristics of dielectric barrier discharge plasma actuators," in Proceedings of 2013 European Radar Conference (EuRAD), Nuremberg, Germany, 2013, pp. 69-72.

[11] M. Born and E. Wolf, Principles of Optics: Electromagnetic Theory of Propagation Interference and Diffraction of Light. Cambridge, UK: Cambridge University Press, 1999.

[12] D. K. Cheng, Field and Wave Electromagnetics, 2nd ed. Reading, MA: Addison-Wesley Publishing, 1989.

[13] Y. Itikawa, "Effective collision frequency of electrons in gases," The Physics of Fluids, vol. 16, no. 6, pp. 831-835, 1973.

[14] A. Srivastava, G. Prasad, P. K. Atrey, and V. Kumar, "Attenuation of microwaves propagating through parallel-plate heli-

\section{Yuna Kim}

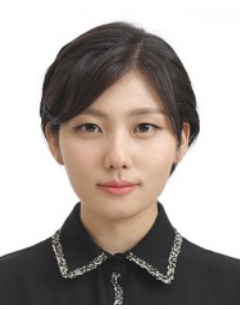

received her B.S. degree in electrical and electronic engineering from Yonsei University, Seoul, Korea, in 2012. She is currently working toward her Ph.D. degree in electrical and electronic engineering at Yonsei University. Her current research interests are plasma analysis, HEMP coupling, and numerical analysis based on multi-physics including thermo-dynamics and electromagnetics. Her recent research focuses on electric problems caused by high temperatures in a circuit.

\section{Sangin Kim}

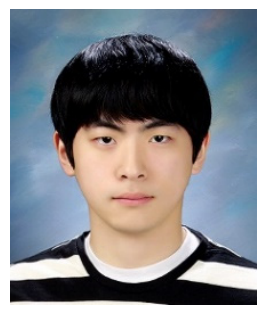

received his B.S. degree in electrical and electronic engineering from Yonsei University, Seoul, Korea, in 2015. He is currently working toward his Ph.D. degree in electrical and electronic engineering at Yonsei University. His current research interests are plasma analysis, HEMP, electromagnetic shielding analysis, vital sign sensor, and radio frequency system. His recent research focuses on electric problems caused by HEMP.

\section{Doo-Soo Kim}

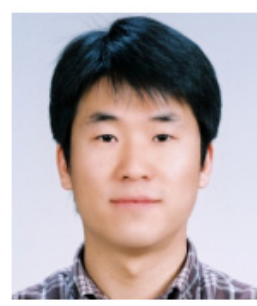

was born in Gwangju, Korea. He received his B.S. degree from Sogang University, Seoul, Korea, in 2001 and his M.S. degree in electrical and electronic engineering from POSTECH, Pohang, Korea, in 2006. He currently works for the Agency for Defense Development, Daejeon, Korea, as a senior researcher. um glow discharge at atmospheric pressure," Journal of Applied Physics, vol. 103, no. 3, article ID. 033302, 2008.

[15] K. Samanta, M. Jassal, and A. K. Agrawal, "Atmospheric pressure glow discharge plasma and its applications in textile," Indian Journal of Fibre and Textile Research, vol. 31, no. 1, pp. 83-98, 2006.

[16] Y. B. Suzen and P. G. Huang, "Simulations of flow separation control using plasma actuators," in Proceedings of the 4th ALAA Aerospace Sciences Meeting and Exbibit, Reno, NV, 2006.

[17] C. L. Enloe, T. E. McLaughlin, R. D. Van Dyken, K. D. Kachner, E. J. Jumper, T. C. Corke, M. Post, and O. Had$\mathrm{dad}$, "Mechanisms and responses of a single dielectric barrier plasma actuator: geometric effects," ALAA Journal, vol. 42, no. 3, pp. 595-604, 2004.

Il-Young Oh

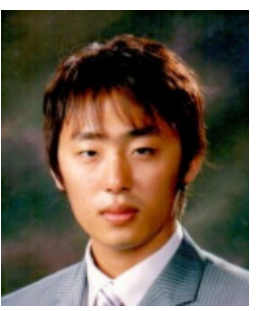

received his B.S. and Ph.D. degrees in electrical and electronic engineering from Yonsei University, Seoul, Korea in 2007 and 2013, respectively. He is currently a professor at the School of Electrical Engineering, Dongyang Mirae University, Seoul, Korea. His main research interests are numerical analysis based on the low dispersion finite difference time-domain method, plasma analysis, and HEMP coupling.

Jong-Gwan Yook

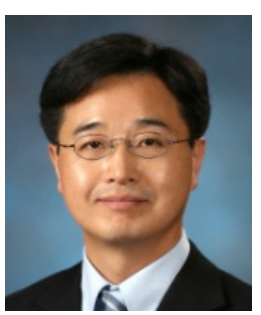

was born in Seoul, South Korea. He received his B.S. and M.S. degrees in electronics engineering from Yonsei University, Seoul, South Korea, in 1987 and 1989, respectively, and his Ph.D. degree from the University of Michigan, Ann Arbor, MI, USA, in 1996. He is currently a professor at the School of Electrical and Electronic Engineering, Yonsei University, Seoul, Korea. His main research interests are theoretical/numerical electromagnetic modeling and characterization of microwave/millimeter-wave circuits and components, design of radio frequency $(\mathrm{RF})$ integrated circuits and monolithic microwave integrated circuits, and analysis and optimization of high-frequency high-speed interconnects, including signal/power integrity, based on frequency as well as time-domain full-wave methods. Recently, his research team developed various biosensors, such as carbon nanotube RF biosensors for nanometersized antigen-antibody detection and remote wireless vital signal monitoring sensors.

His current research interests include active phased array antenna systems and optimization algorithms. 